\section{o Caveat Emptor: The Perils of Panel Testing in Hereditary Breast Cancer}

\section{TO THE EDITOR:}

In a recent issue of Journal of Clinical Oncology, Beitsch et al ${ }^{1}$ argue for expanded panel testing in all patients with a diagnosis of breast cancer. Their argument rests on the finding that of patients who met National Comprehensive Cancer Network guidelines, 9.39\% had a pathogenic/likely pathogenic (P/LP) variant, whereas of those patients who did not meet National Comprehensive Cancer Network guidelines, $7.9 \%$ had a P/LP variant. These figures, however, include many variants in genes that have no definite proven association with an increased risk of breast cancer. ${ }^{2}$ These results may thus represent incidental findings that could equally be found in an unselected population and will not inform the management of their disease. Indeed, even at below the expected carrier frequency of one in 50, monoallelic variants in the recessively inherited gene MUTYH account for a full $20 \%$ of the reported findings in patients who did not meet NCCN guidelines. These findings are not pertinent and amount to population screening.

Reanalysis of the data including only variants in genes with definitive evidence for breast cancer susceptibility ${ }^{3}$ determines that of patients who met NCCN guidelines, $6.47 \%$ had a P/LP variant, whereas of those patients who did not meet guidelines, 3.75\% had a P/LP variant (Fig 1). Not surprisingly, as NCCN guidelines are intended to identify patients who are at high risk, the majority of variants identified in patients

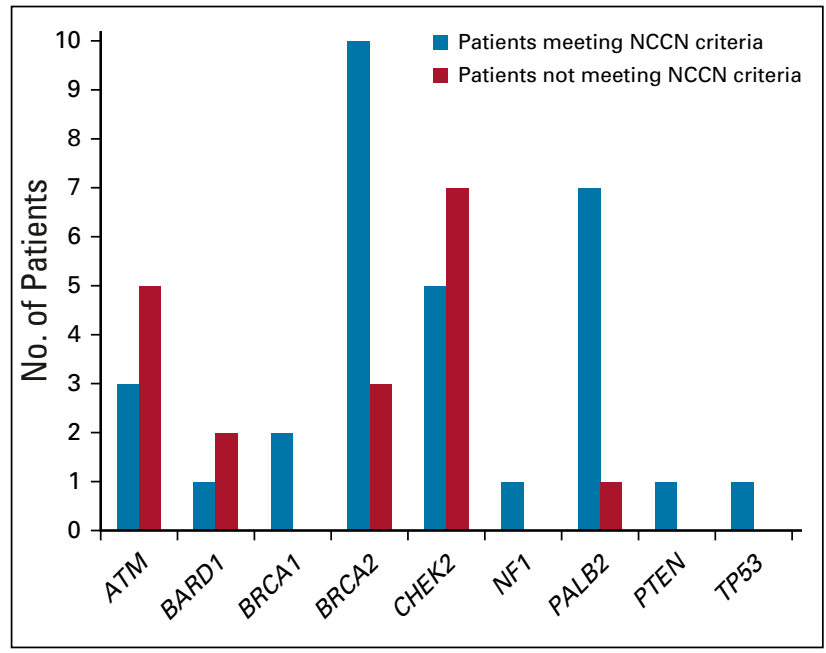

FIG 1. Selected data from Beitsch et al, ${ }^{1}$ including only genes with definitive evidence for breast cancer susceptibility. NCCN, National Comprehensive Cancer Network. who did not meet NCCN guidelines were in the moderate-risk genes ATM, BARD1, and CHEK2, which do not affect surgical management. Even the inclusion of $B A R D 1$ as a proven breast cancer gene is debatable as it is not significantly overrepresented in cases versus controls, ${ }^{4}$ and a significant increase in breast cancer risk has not been demonstrated. ${ }^{5}$ Testing using NCCN guidelines missed four P/LP variants in high-risk genes, three in $B R C A 2$, and one in PALB2. This represents $0.4 \%$ of the tested population.

The cost of indiscriminate expanded panel testing, aside from the financial implications, lies in the return of variants of uncertain significance (VUSs) to health care providers with limited understanding of their meaning. In the study by Beitsch et al, ${ }^{1}$ the $54 \%$ of patients who were found to have VUSs is unacceptably high. These results take time to interpret and explain to patients and may require follow up, additional testing, or review in case of reclassification. ${ }^{6}$ Of even greater concern, results are frequently misinterpreted, leading to inappropriate clinical management. Kurian et $\mathrm{al}^{7}$ found that many surgeons managed patients with BRCA1/BRCA2 VUSs in the same manner as patients with BRCA1/ BRCA2 pathogenic mutations, and one half of average-risk patients with VUSs underwent bilateral mastectomy, which suggests a limited understanding of results among both surgeons and patients. The recognized shortage of genetic health professionals ${ }^{8}$ means that there is no short-term solution to this issue, but the VUS rate can be minimized by restricting testing to genes that are clinically relevant to the patient's presenting diagnosis. ${ }^{9}$ In the longer term, functional studies of genes and variants, as well as population-level data with accurate phenotyping, will improve variant classification and reduce uncertainties. ${ }^{6}$

Lastly, we note that at least one third of the 27 authors of the work by Beitsch et $\mathrm{al}^{1}$ are employees of or receive honoraria, research funding, or indirect support from diagnostic laboratories that are heavily involved in marketing gene panels, which could result in a significant conflict of interest when interpreting of the results of the study.

\section{Amy Taylor, PhD}

Cambridge University Hospitals National Health Service Trust, Cambridge, United Kingdom

\section{Marc Tischkowitz, MD, PhD}

Cambridge University Hospitals National Health

Service Trust and University of Cambridge, Cambridge, United Kingdom 
AUTHORS' DISCLOSURES OF POTENTIAL CONFLICTS OF INTEREST AND DATA AVAILABILITY STATEMENT

Disclosures provided by the authors and data availability statement (if applicable) are available with this article at DOI https://doi.org/10.1200/ JCO.19.00122

\section{REFERENCES}

1. Beitsch PD, Whitworth PW, Hughes K, et al: Underdiagnosis of hereditary breast cancer: Are genetic testing guidelines a tool or an obstacle? J Clin Oncol 37:453-460, 2019

2. Easton DF, Pharoah PD, Antoniou AC, et al: Gene-panel sequencing and the prediction of breast-cancer risk. N Engl J Med 372:2243-2257, 2015

3. Lee K, Seifert BA, Shimelis $H$, et al: Clinical validity assessment of genes frequently tested on hereditary breast and ovarian cancer susceptibility sequencing panels. Genet Med 10.1038/s41436-018-0361-5 [Epub ahead of print on December 3, 2018]

4. Thompson ER, Rowley SM, Li N, et al: Panel testing for familial breast cancer: Calibrating the tension between research and clinical care. J Clin Oncol 34:1455-1459, 2016
5. Li J, Meeks H, Feng BJ, et al: Targeted massively parallel sequencing of a panel of putative breast cancer susceptibility genes in a large cohort of multiple-case breast and ovarian cancer families. J Med Genet 53:34-42, 2016

6. Hoffman-Andrews L: The known unknown: The challenges of genetic variants of uncertain significance in clinical practice. J Law Biosci 4: 648-657, 2018

7. Kurian AW, Li Y, Hamilton AS, et al: Gaps in incorporating germline genetic testing into treatment decision-making for early-stage breast cancer. J Clin Oncol 35:2232-2239, 2017

8. Feldman GL: 2016 ACMG Annual Meeting presidential address: The practice of medical genetics-Myths and realities. Genet Med 18:957-959, 2016

9. Taylor A, Brady AF, Frayling IM, et al: Consensus for genes to be included on cancer panel tests offered by UK genetics services: Guidelines of the UK Cancer Genetics Group. J Med Genet 55:372-377, 2018

DOI: https://doi.org/10.1200/JC0.19.00122; Published at jco.org on June 27, 2019. 


\section{Caveat Emptor: The Perils of Panel Testing in Hereditary Breast Cancer}

The following represents disclosure information provided by authors of this manuscript. All relationships are considered compensated. Relationships are self-held unless noted. I = Immediate Family Member, Inst = My Institution. Relationships may not relate to the subject matter of this manuscript. For more information about ASCO's conflict of interest policy, please refer to www.asco.org/rwc or ascopubs.org/jco/site/ifc.

No potential conflicts of interest were reported. 